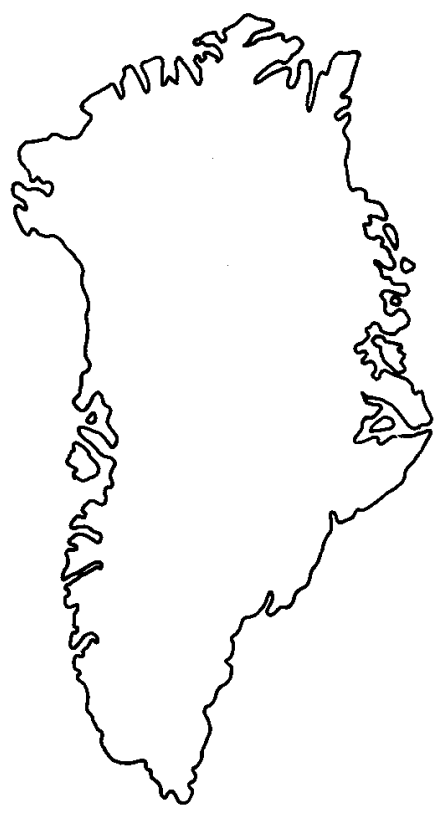

\title{
Geologic photogrammetry using standard small-frame cameras
}

\author{
Keld S. Dueholm
}

\begin{abstract}
Multi-model photogrammetry enables precise three-dimensional measurements from strips or blocks of overlapping small-frame photographs (colour slides). The method can be used for geo-scientific terrain analysis and mapping. Of special interest is the ability to map otherwise inaccessible terrain features such as geological outcrops on steep mountain faces and canyon walls. Field photography is carried out without special photogrammetric training using ordinary small-frame cameras. Photographs can be taken at any scale and angle from terrestrial stations, helicopters, light planes, or boats. In the laboratory, strips or blocks of small-frame photographs are set up in an analytical stereo-plotter where multiple stereoscopic model pairs are simultaneously orientated. Interpretation and compilation is continuous across model boundaries. Data can be plotted in many different projections.

The multi-model photogrammetric technique is explained and procedures are outlined for camera calibration, photography, and acquisition of ground-control information.
\end{abstract}

K. S. D., Institute of Surveying and Photogrammetry, Technical University of Denmark, DK-2800 Lyngby, Denmark.

Photogrammetry is used primarily as a technique to produce precise topographic maps from aerial photographs. The method has also been applied for many years to geologic photo-interpretation and mapping at the U.S. Geological Survey and the Geological Survey of Greenland (Pillmore et al, , 1981). In the traditional photogrammetric method, overlapping vertical aerial photographs are set up in a mechanical photogrammetric plotting instrument, creating a stereoscopic model. When properly orientated, this model is a true, deformation-free representation of the photographed terrain. By means of a tracing assembly, the operator controls the three-dimensional movements of a floating mark superimposed on the perceived stereoscopic model. Using the floating mark as a pointing device outcrops be can traced, maps compiled, and precise three-dimensional measurements can be made.

Pre-existing vertical aerial photographs, originally taken for topographic mapping purposes, are used for conventional geologic photogrammetry. In many projects these large-frame, black and white exposures provide an excellent basis for map compilation, measurement of structural parameters, and storing and manipulating three-dimensional surfaces (Dueholm \& Pillmore, 1989; Hougaard et al., 1991). However, the resolution is often inexpedient for the purpose of other geological mapping projects. An inconvenient scale, a poor angle of view, or shadows in the photographs may obscure critical detail, making them extremely hard to identify and follow with the floating mark of the photogrammetric instrument. If the vertical aerial photographs are of a steep feature, such as a mountain side, then details are often impossible to see and map. Only in very rare situations are photogrammetric flight operations equipped for oblique photography. These problems can now be overcome by applying photogrammetric techniques directly to field photographs, using the new multi-model method (Dueholm, 1990).

The multi-model method uses an analytical stereoplotter for three-dimensional measurements and map compilation. The analytical plotter is a computer-controlled photogrammetric instrument in which the stereoscopic model is controlled by mathematical formulas and parameters within the computer software. Because the stereoscopic model is maintained mathematically instead of mechanically, virtually all types of photographs can be accommodated by the software. The multi-model software programme enables the simultaneous set-up of strips or blocks of small-frame photographs that form multiple stereoscopic models. Models are set up alone or in combination with aerial photographs. The method then allows for the free 
movement between the aerial model and the smallframe model(s) during subsequent interpretation and mapping.

\section{The multi-model photogrammetric method}

\section{Field Photography}

Strips or blocks of overlapping photographs are taken from successive positions along a terrain feature. The photographs are taken according to guidelines outlined in the section 'Photography' (see below). Knowledge of the camera station positions or angle of rotation between frames is not required. Therefore, photographs can be taken obliquely out of the open window of a helicopter or light aeroplane flying along a mountain side, or from a boat sailing along steep coastal cliffs. The camera used for photography can be the typical small-frame field camera carried by geologists on expeditions. Any stable camera with a good quality lens that has manual controls or fixed focus can be used, but it must be calibrated either before or after the field photographs have been taken. Camera requirements and a camera calibration procedure are described in the next section.

As an example, Fig. 1 shows a hypothetical photography situation. An area is covered by pre-existing pan- chromatic vertical aerial photographs originally used for topographic mapping. These photographs are subsequently used for geological photo-interpretation and mapping, but a steep coastal mountain face does not appear with sufficient resolution for geological study. Therefore, on a reconnaissance flight by helicopter, the pilot is directed to fly along the mountain face at a suitable height and distance from the mountain. Through the open window of the helicopter the geologist takes overlapping photographs with his smallframe camera. A near vertical coastal cliff of special interest requires more detailed analysis. For this study the geologist motors along the cliff in a rubber dinghy for close range photography.

\section{Multi-model blocks}

In the laboratory, the small-frame photographs are grouped in strips or blocks, called multi-model blocks, that fit the two stage plates of the analytical plotter. The stage plates will each hold 20 photographs taken with 35 $\mathrm{mm}$ cameras or 9 photographs taken with $70 \mathrm{~mm}$ cameras. Therefore, as many as 39 models formed by overlapping $35 \mathrm{~mm}$ photographs or 17 models formed by overlapping $70 \mathrm{~mm}$ photographs can be set up simultaneously. In order to facilitate the handling of many small-frame photographs, alternating left and right pho-

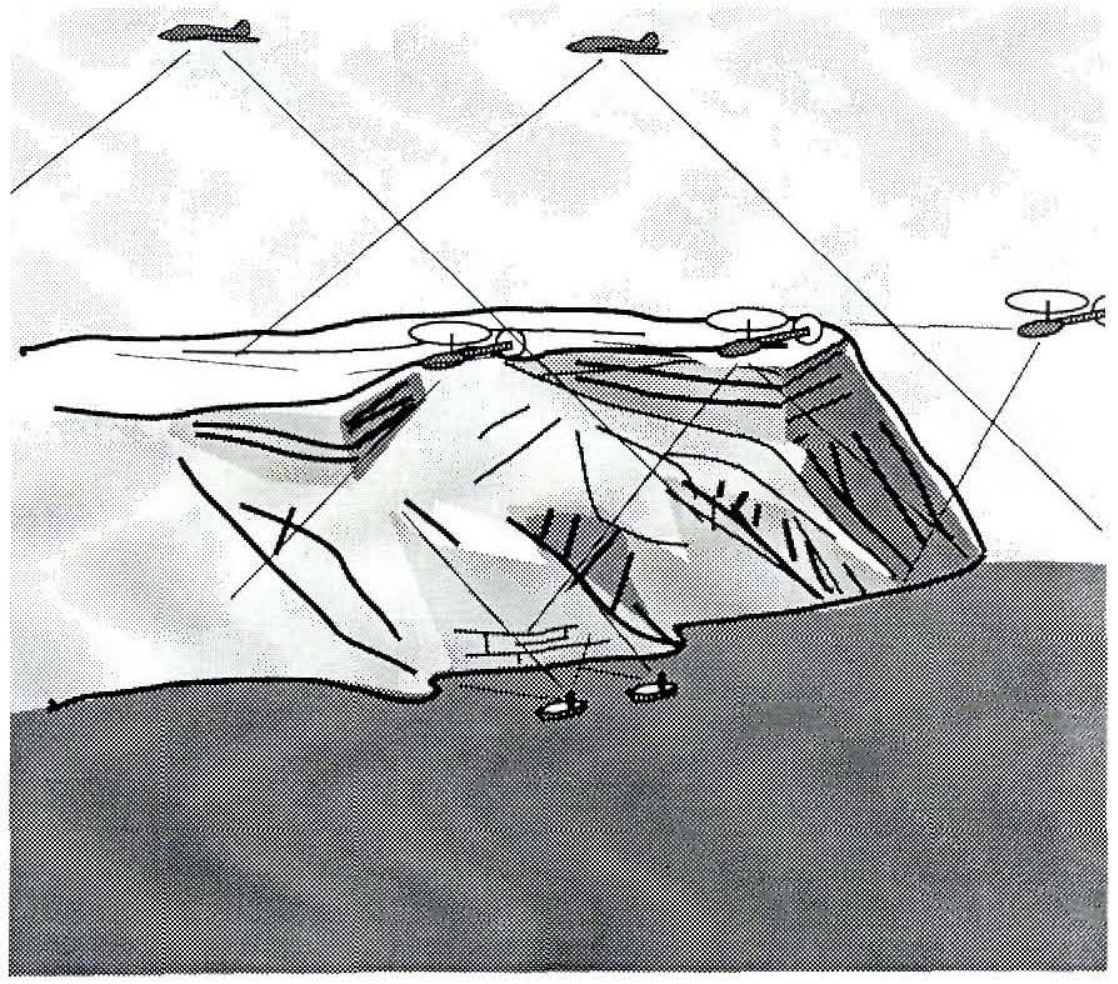

Fig. 1. Sketch by O. Jacobi showing hypothetical photography of steep coastal cliffs. Stereo photographs are taken from a helicopter and close up images from a rubber dinghy. In the multimodel method these photographs are combined with pre-existing vertical aerial photographs, indicated by the aeroplanes in the sketch. Thus, the cliffs can be studied photogrammetrically in the different scales and view angles of the various photographs. 

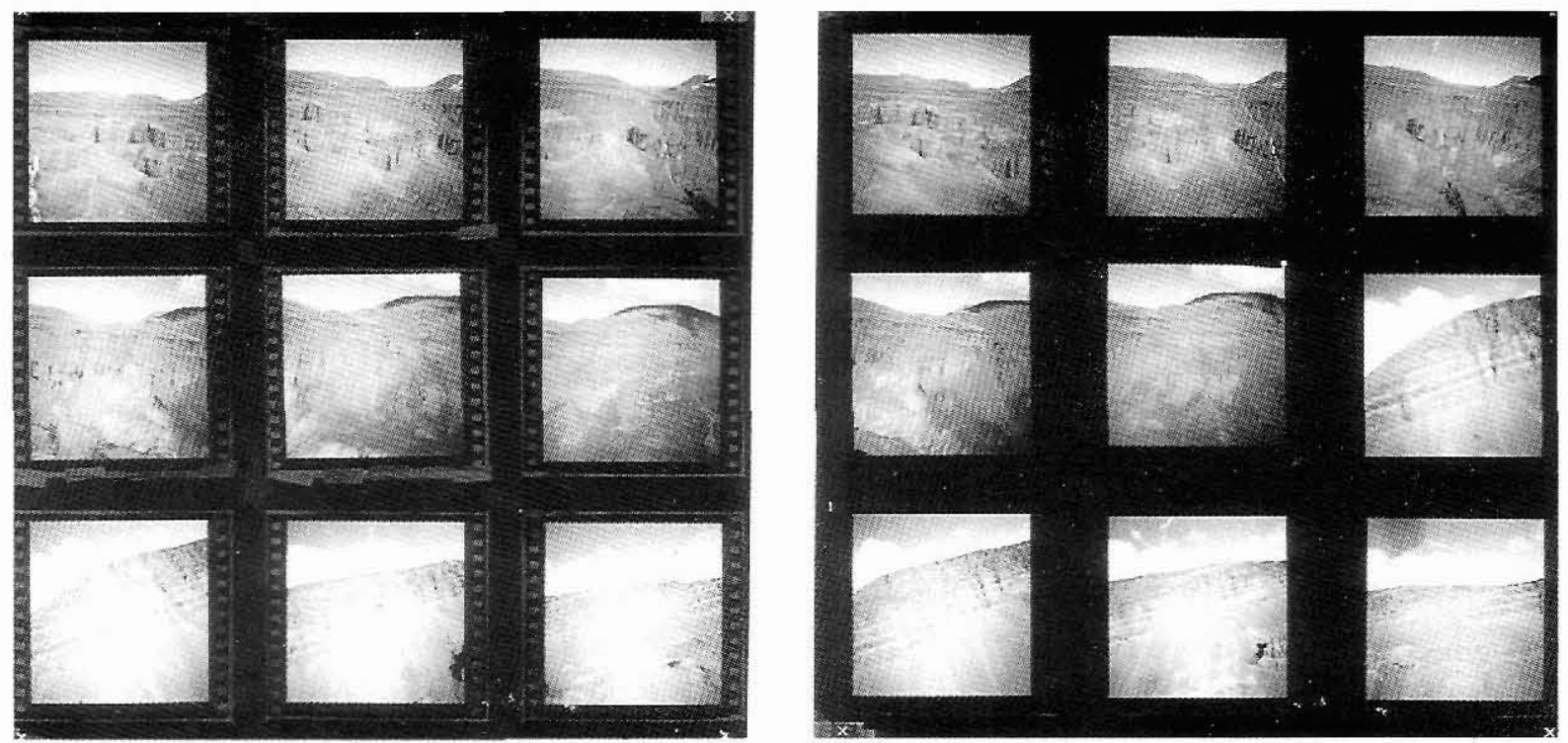

Fig. 2. A template set where small-frame photographs (originals in colour) taken with a Hasselblad camera are mounted in holes cut out of two film sheets. Alternating left and right photographs of the model pairs are mounted on the two templates. The photographs constitute two multi-model blocks. The top eleven photographs (six on the left template and five on the right) form a strip of ten stereoscopic models. The remaining seven photographs form a separate strip of six models. The white crosses at the template corners are fiducial marks.

tographs of the model pairs can be contact printed onto two film sheets called templates (Fig. 2), one for each stage plate (Dueholm, 1990). If equipment for contact printing is not readily available, templates can be made by mounting the original small-frame photographs in holes cut-out of mylar or other stable film sheets.

Photographs of different origin, scale, and angle of view can be set up in one multi-model block. In the example from Fig. 1 a model formed by two aerial photographs can be set up together with selected models formed by the small-frame photographs. Thus, in one multi-model block the geologist can work in the general, birds-eye view of the vertical aerial photographs, the oblique view of the helicopter colour photographs, and the close up view of the boat photographs covering the vertical cliff at a large scale.

In the case of combining aerial photographs and small-frame photographs, the small-frame photographs can be mounted in holes cut out of those parts of the aerial photographs that are not required for the current model (Fig. 3). Thus, the aerial photographs can serve as templates for small-frame photographs, and at the same time provide a stereoscopic model themselves.

\section{Ground control}

All photogrammetric mapping methods require the use of ground control for scaling and levelling of the stereoscopic model. However, a method which requires that control points are surveyed in the field is of little benefit to geologists. In the multi-model technique, control points required for orientation of the stereoscopic models are transferred from topographic maps or vertical acrial photographs.

For the orientation of the vertical aerial photographs in Fig. 1 aerotriangulated control points may be available from a topographic survey, or alternatively the models may be set up with reference to features on detailed modern topographic maps. The strip of models formed by the oblique photographs taken from a helicopter are then tied to the vertical aerial photographs using points identified and measured in both the vertical and oblique photographs. The strip of models formed by the close up boat photographs is similarly tied to the oblique helicopter photographs.

Simple measurements (such as tape distances, compass directions, or altimeter elevations) taken in the field can also be used for orientation. Control information is further described in the section entitled 'Ground control'.

\section{Multi-model orientation}

The multi-model software programme is used to set up the templates in the analytical plotter and orientate the models. The programme performs a bundle block 

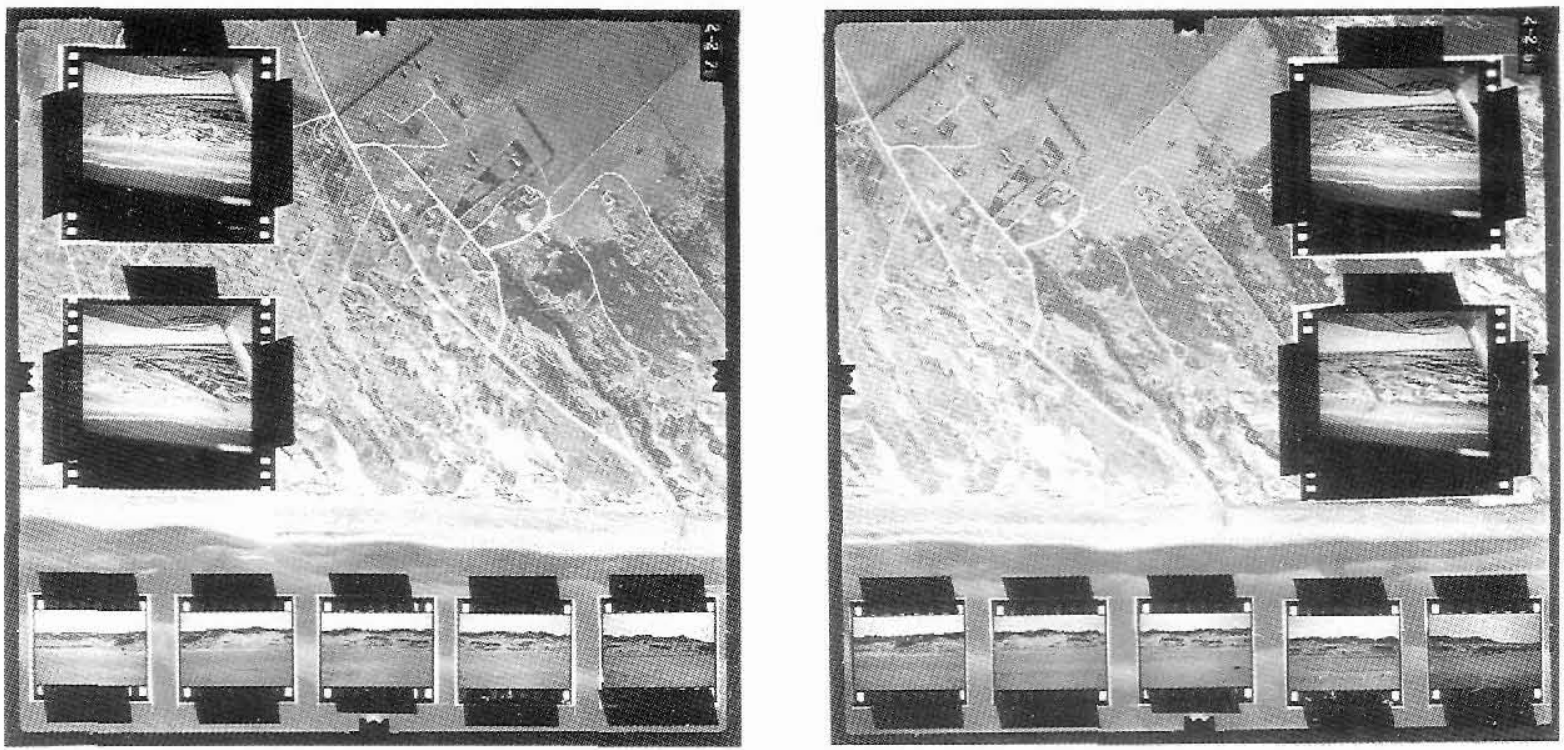

Fig. 3. A multi-model block where small-frame photographs are mounted in holes cut out of vertical aerial photographs. The vertical acrial photographs thus serve as templates for the small-frame photographs, and at the same time provide a stereoscopic model themselves in the uncut areas. The multi-model block consists of thirteen stereoscopic models: one model formed by the overlapping acrial photographs, a strip of three models formed by the four 70 - $\mathrm{mm}$ photographs, which were taken from a helicopter, and a strip of nine models formed by the ten $35-\mathrm{mm}$ images taken from terrestrial stations. The four fiducial marks used for orientation of each of the two vertical aerial photographs with insets are located on their sides.

adjustment of the ground control information and photogrammetrically measured tie points between photographs. The operator is guided through the measurement of tie points by the programme. Transfer of tie points between photographs is based on stereoscopic identification. No marking or description of points is needed within one set of templates. During the bundle block adjustment, all observations (tie points and ground control) are weighted by the inverse square of an a priori error provided by the operator. Preliminary values for the adjustment parameters are automatically calculated. The programme includes automatic identification of gross errors and reduction of weight on the observations involved.

\section{Resetting of multi-model blocks}

The template sheets (Fig. 2) or the aerial photographs (Fig. 3) that hold the small-frame photographs each have four tick marks (fiducial marks), that are used to register the block of photographs on a template set to the stage plates of the analytical plotter. When a multimodel block has been set up the first time, which is time consuming, the parameters of the bundle block adjustment are stored in data files. Resetting of the block is done by simply measuring the four tick marks of each of the two templates involved. This procedure takes only a few minutes. Therefore, templates already prepared, orientated, and archived can be reset at any time and used for additional interpretation and compilation. Because of the very fast resetting procedure, several different multi-model blocks can be conveniently used for compilation during a work day.

\section{Geological mapping}

After the set up of the photographs in the analytical plotter is completed, the geologist can proceed with geological interpretation and mapping using GEOPROGRAM (Dueholm \& Coe, 1989). This programme is used to measure geological structures and to draw maps and sections and axionometric or perspective views, and it supports three-dimensional analysis and data collection continuously across model boundaries. The geologist views the images through stereoscopic optics and guides the floating mark through the models. An automatic model change occurs whenever the floating mark is moved across the image frame line of either of the two photographs of the current model. The floating mark moves to the same point in the neighbouring model across the frame line. In addition, the model can be changed by command. In this way the floating mark 
may be moved to the same point in models formed by photographs of different scale or view angle within the same template set.

The analytical plotter monitors the $X, Y$, and $Z$ coordinates of the floating mark. Three-dimensional coordinates can therefore be digitised into computer memory. Using GEOPROGRAM, the geologist can collect and manipulate three-dimensional data digitised from the stereo photographs, calculate geological parameters (such as, strike and dip of bedding, plunge and direction of fold axes, and stratigraphic thickness of beds), and project planes that represent geological surfaces into the stereoscopic model which allows visual extrapolation from, and interpolation between, measured outcrops. The programme also includes facilities for storing, retrieving, plotting, and displaying lines, symbols, and text. Line types and symbols can be defined individually and data can be labelled.

\section{Plot projections}

During map compilation or off-line plotting of recorded data, several plot projections can be defined and used. Currently, the following plot projections are available: (1) orthographic projection, that allows definition of any plane on which to project data by describing the plane's direction and tilt (equivalent to strike and dip angles of geologic planes) thereby generating normal map projections and sections plotted onto oblique or vertical profile planes; (2) perspective views and isometric or diametric drawings, that can be seen from any direction and at any angle; and (3) full periphery projection, that is used for displaying maps of tunnel and shaft walls.

A switch between defined projections during map compilation can be made by merely entering an identification number. The capability of plotting in several projections allows the inspection and evaluation of the compiled data from many angles during interpretation and mapping. For example, different projections could produce a traditional orthogonal map, a vertical profile plane, or a perspective view of the data. Because recorded data are stored in three dimensions, they may be subsequently plotted in any available projection.

\section{Data verification}

A GEOPROGRAM function allows the operator to scroll through recorded data. As this is done, the floating mark of the photogrammetric instrument simultaneously moves to the coordinate positions of the data points and the operator can see how each feature was mapped. Foot switches on the analytical plotter control the direction and speed of scroll. In effect, this facility superimposes, in a point-by-point, line-by-line manner, previously measured and recorded data onto the stereoscopic image of the scene. Thus, it provides a means of verifying recorded data or visually locating any threedimensional data that have either previously been mapped and stored in GEOPROGRAM or translated into the GEOPROGRAM-recording format from other data sources.

\section{Accuracy}

The photogrammetric accuracies obtained are better than 20 microns on the photograph scale. For example, a steep mountain face may be photographed from a distance of one kilometre with a $35 \mathrm{~mm}$ mirror-reflex camera using a $35 \mathrm{~mm}$ wide angle lens. This gives photographs on a scale of 1:30 000 and potential accuracy better than $60 \mathrm{~cm}$. For very detailed studies, in for example quarries, photographs taken at a distance of 10 $\mathrm{m}$ with the same camera would give a photograph scale of c. 1:300 and measurement accuracy better than 6 $\mathrm{mm}$. Absolute accuracy depends on the ground control situation. Ground control of superior accuracy will provide absolute accuracy equal to the photogrammetric accuracy. Often, one has to use less accurate ground control (see the section entitled 'Ground control'), and here the absolute accuracy decreases to the accuracy of the ground control. However, since the ground control information is weighted during bundle block adjustment, the relative accuracy between models remains equal to the photogrammetric accuracy.

\section{Instrumentation}

The multi-model method was developed for the Kern DSR 15 analytical plotter with a DEC VMS operating system. Prototypes of the new multi-model photogrammetric system are available from the Institute of Surveying and Photogrammetry, Technical University of Denmark and the Digital/Photogrammetric Geologic Mapping Laboratory (Plotter Lab), U.S. Geological Survey in Denver.

\section{The camera}

Camera parameters, such as the lens distortion, the precise focal length and the principal point (position of the image relative to the lens), must be known in photogrammetry. Standard small-frame cameras generally have large lens distortion and variable focal length that do not have the precision necessary for accurate photogrammetry. However, the multi-model programme is 
able to calculate these parameters using one of several methods: (a) camera parameters are calculated during the set-up of an individual model, or block of models, by using ground-control points; (b) camera parameters are determined by camera calibration and then used as known parameters during the subsequent set-up of models; or (c) camera parameters are calculated using any combination of methods (a) and (b). Method (a) requires accurate, well distributed ground control. Because ground control is normally difficult to acquire, method (b) is often the most feasible in geologic photogrammetry. Method (c) is feasible when a camera has been calibrated at a different focal length than that required for a particular project. In this case, the distortion curve and the principal point can be obtained from the camera calibration, whereas the unknown focal length is calculated separately using at least one additional control point in the model set-up.

\section{Camera calibration}

A calibration-point array (test field) and the multimodel programme are used for camera calibration. The test field consists of about one hundred targets equally distributed in a spatial steel framework. The targets are surveyed using electronic theodolites to an accuracy of $0.1 \mathrm{~mm}$ standard deviation. The framework is built so that no deformation, other than an overall scale change (unimportant for camera calibration) results from changes in temperature.

To calibrate a camera, the test field is photographed from the front, so that the target array fills the entire image. The camera must be set at the same focus as it will be set for subsequent photogrammetric use in the field. Focusing at infinity is appropriate for most geologic applications. In order to reproduce the calibrated focal length precisely, the focal ring is turned until it stops at infinity. If a closer focus is required, the focal adjustment should be fixed (taped) at the required focus position and not changed between calibration and use in the field.

The developed film is mounted in the analytical plotter and the coordinates of the targets are measured in the image. The multi-model programme compares the measured photograph coordinates with the results of the target survey using a self-calibrating bundle adjustment. In this way it calculates the camera position and rotation as well as the best-fit lens distortion curve parameters, focal length, and principal-point coordinates.

Preferably, calibration should be performed before field work in order to determine the stability of the camera. However, the calibration data are not required during the actual field photography; if the camera is known to be stable, calibration after field work is acceptable.

\section{Fiducial marks and film flatness}

There are two remaining difficulties concerning the use of calibrated standard cameras for photogrammetry:

1) The camera lacks fiducial marks or reseau crosses that can define an image coordinate system. Therefore, the coordinate measurements must be based on the establishment of the corners of the photograph or the frame lines. Commonly the photograph corners are rounded and the shape differs slightly from frame to frame because of film warp. An easy and sufficiently precise way to measure a corner of a photograph is to visually extrapolate the frame lines to their intersection, and measure this intersection point.

2) The camera does not have pressure or vacuum devices to keep the film flat against the camera back. Present experience shows that $35 \mathrm{~mm}$ film is less influenced by film warp than $70 \mathrm{~mm}$ film due to the smaller camera frame that reduces the free film span in the 35 mm camera.

As a result of these difficulties, standard small-frame cameras are expected to give a somewhat lower accuracy than metric cameras with fiducial marks or reseau crosses and vacuum or pressure backs. However, many standard cameras have been tested with very good results. The reduction of accuracy encountered has been negligible, considering the accuracy demands of most geological mapping studies.

\section{Metric cameras}

If accuracy is a concern, metric cameras are available that can be used for hand-held photography (e.g. Hasselblad MKWE, Pentax PAMS645 and Rolleimetric 6006). These metric cameras are relatively expensive and heavy to carry.

\section{Photography}

Three-dimensional coordinate positions can be calculated from measurements on any pair of overlapping photographs provided the two photographs are taken from different camera stations. However, the accuracy of the calculated coordinates and the perception of the stereoscopic model depends on the geometry of the photographing situation.

Stereoscopic perception of the model is important for interpretation and measurement. In industrial and ar- 


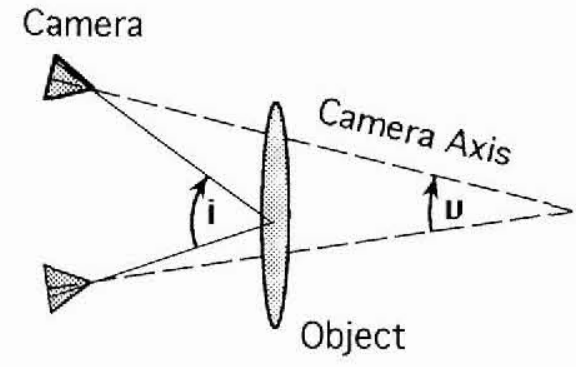

Fig. 4. Sketch, viewed from above, illustrating the taking of stereo photographs. The convergence angle $(v)$ of the camera axes and the intersection angle (i) of light rays connecting object points with the camera stations are shown.

chitectural photogrammetry, monoscopic measurements, i.e. measurement in two orientated photographs but without stereo vision, are possible when the object being measured is well defined and easy to identify in both photographs. This is almost never the case with geologic features. Stereoscopic perception depends on the convergence angle $(v)$ between the axes of the two cameras used (Fig. 4). When the convergence angle exceeds $20^{\circ}$ stereoscopic viewing becomes very difficult. The best stereoscopic perception is obtained when the camera axes are parallel $(v=0)$.

The accuracy of the calculated depth coordinate (in the direction of the camera axis) is dependent on the angle of intersection (i) between the two light rays that connect the point of interest in the scene with the camcra lenses of the two photographing stations (Fig. 4). A right angle intersection gives the best result. The accuracy of the depth coordinate decreases as the intersection angle varies from a right angle. Therefore, until the intersection angle exceeds $90^{\circ}$, the further apart the camera stations, the more accurate the results.

\section{Taking parallel photographs}

Photographs for photogrammetric use are normally taken so that camera axes are parallel and images overlap about 60 percent. In this way, each stereoscopic model pair overlaps a neighbouring pair by about 20 percent of the frame size. This ensures stereoscopic coverage even if the camera stations and photograph directions vary somewhat from those that were planned.

The distance $(B)$ between camera stations (the photo base) is calculated from the camera-to-object distance $(D)$, the focal length $(c)$, the frame size $(f)$, and the overlap (Fig. 5) according to the following formula:

$$
B=(1-0.6) D f / c
$$

An overlap of 60 percent is used in the formula. The camera-to-object distance is calculated from the desired average photograph scale $(S)$ by means of the formula:

$$
D=c / S
$$

The camera-to-object distance should be the shortest distance from the camera to any point of interest in the scene. Stereoscopic coverage is then ensured for all other parts of the scene.

Excessive depth variations in the scene can make stereoscopic perception difficult when viewing photographs that only overlap 60 percent. In addition, 60 percent overlap might not always ensure sufficient stereoscopic coverage when the camera is hand held. Therefore, photographs should be taken with 80 percent overlap in the field (photo basis equal half the calculated $B$ ). Later, during stereoscopic mapping, the 60 percent overlap of alternating photographs may be sufficient for the majority of models.

Wide-angle lenses should be used to obtain a reasonable depth accuracy (large intersection angles). Focal lengths no longer than $35 \mathrm{~mm}$ for $35 \mathrm{~mm}$ cameras and no longer than $60 \mathrm{~mm}$ for $70 \mathrm{~mm}$ cameras are recommended. With both types of cameras and 60 percent overlap, the accuracy of depth measurement is about half the accuracy of measurements in planes that are parallel to the photo plane. With increasing focal length and/or overlap, the intersection angle decreases to a point where it will be difficult to orientate the photographs properly in the analytical plotter.

\section{Helicopter photography}

Present logistic work procedures in Greenland use helicopter transport for the geologist's camp changes and reconnaissance flights. Helicopters are excellent

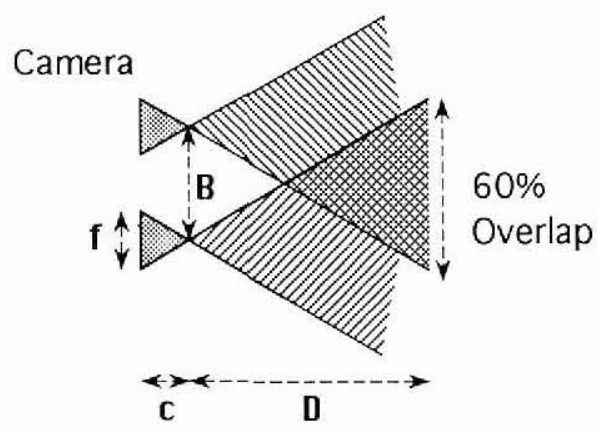

Fig. 5. Sketch, viewed from above, illustrating the taking of parallel photographs with 60 percent overlap. Parameters involved in the computation of the photo basis $(B)$ are shown. 
platforms for oblique photography of steep mountain sides. The following procedure is recommended when taking photographs from a helicopter (or a light aeroplane).

For best stereoscopic perception the camera is pointed at the slope of interest so that the image plane is approximately parallel to this slope. The pilot is directed to fly along straight lines or in very gentle curves. For each sharp turn the helicopter must circle and start a new photographic strip. The pilot controls the flight height using the altimeter of the helicopter, but the distance to the mountain side is established by the geologist who takes the photographs. Before a photographic strip is started the geologist directs the pilot to the correct distance. This is done by evaluating the extent of the scene in the view finder of the camera while flying towards the scene at the required flight height. The pilot will normally be able to navigate at this approximate distance during the photography of the entire strip.

The photo basis $(B)$ or time lap between exposures is best judged in the view finder. When a photograph is taken the central point of the scene is observed and remembered. While keeping the camera in the same direction the central point is followed continuously in the view finder and the next photograph is taken when the central point has moved $20 \%$ of the frame width (for $80 \%$ overlap).

At the planning stage estimates can be made of the camera-to-object distance, flight height, photo basis, and time lapse between exposures that can serve as guidelines during photography. Formula (2) is used to calculate the camera-to-object distance $(D)$ from the planned photograph scale. An approximate flight height $(H)$ is computed from $D$, an estimated average slope angle $(\emptyset)$ of the scene, and the elevation of the terrain point at the centre of the photograph $(Z)$ (see Fig. 6) by means of the formula:

$$
H=Z+D \cos (\varnothing)
$$

Sufficiently precise values for $Z$ and $\varnothing$ are measured on a topographic map. Photo basis is calculated from formula (1) and by using an anticipated ground speed of the helicopter, the approximate time lapse between exposures may be calculated. The time lapse $(t)$ in seconds is calculated from the ground speed $(G)$ in $\mathrm{km} / \mathrm{h}$ and the photo basis $(B)$ in $\mathrm{m}$ using the following formula:

$$
t=3.6 \mathrm{~B} / \mathrm{G}
$$

As an example a $35 \mathrm{~mm}$ camera with a focal length of 35 $\mathrm{mm}$ is used for photography at a planned scale of $1: 20000$. Using formula (2) $D$ is calculated to $700 \mathrm{~m}$. B

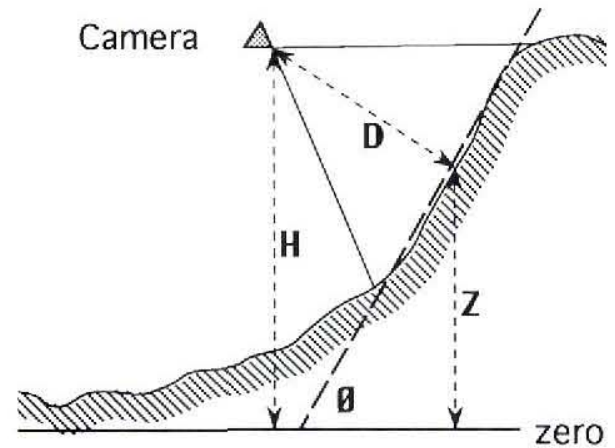

Fig. 6. Sketch illustrating oblique photography from helicopter or aeroplane. Parameters involved in the calculation of an approximate flight height $(H)$ are shown.

is calculated by formula (1) to $288 \mathrm{~m}$ for $60 \%$ overlap or $144 \mathrm{~m}$ for $80 \%$ overlap. Anticipating a ground speed of $100 \mathrm{~km} / \mathrm{h}$, which is typical for small helicopters, the time lapse between exposures is calculated by formula (4) to about 5 seconds. The calculated time lapse is a good guideline during photography. However, the distance to the mountain side, the ground speed, and the camera direction invariably change during the helicopter flight . Therefore, the overlap should always be visually checked by tracking the movement of the centre point in the view finder as described above.

The exposure time must be set sufficiently short to minimise image blur caused by image motion due to vibration of the helicopter and the relative movement of the camera with respect to the scene. As an example, using a shutter speed of $1 / 250$ for the photography situation described above results in an image motion of 10 $\mathrm{cm}$ during the exposure due to a ground speed of 100 $\mathrm{km} / \mathrm{h}$. At the photo scale of $1: 2000010 \mathrm{~cm}$ is equal to 5 microns which is an acceptable figure. For photographs on a larger scale a faster shutter speed is required.

There is no time for measurement of light conditions and individual setting of shutter speed during in-flight photography. Therefore an average setting has to be used for the entire strip. Light is measured from the helicopter towards the mountain side to be photographed. It is important to measure the light reflected by the mountain side itself without influence from a bright sky or sea surface. If possible the light is measured at a close distance. If conditions change drastically during photography the helicopter must circle while a new camera setting is found.

\section{Terrestrial photography}

In order to obtain parallel photography from terrestrial camera stations a compass can be used to direct 
the camera to the same bearing for each photograph station. A correct photo basis is established with sufficient accuracy by estimating the overlap in the view finder of the camera.

Another means of ensuring a correct photo basis is to measure the camera-to-object distance. The camera-toobject distance can be measured on a map, by taping or pacing in the field, or, if necessary, estimated. The photo basis is then calculated using formula (1) and parallel photographing axes are established by estimating the desired overlap in the view finder. If the camerato-object distance is estimated, then extra care should be taken in checking the overlap in the camera's view finder.

A shutter speed of $1 / 125$ is recommended for handheld photography. If a tripod is used shutter speed may be decreased so that very high resolution film can be used.

For a strip of photographs along a steep geologic feature, such as a mountain side, parallel camera axes and 60 percent or 80 percent photograph overlap, as described above, are strongly recommended for the best results. When only a single model is required, convergent photographs may be used. The geologist should use formula (1) to calculate the distance between the camera stations, then rotate the camera to the desired convergence. The convergence angle at which both frames cover the same object area is calculated by the formula:

$$
v=\operatorname{Arctan}(0.4 f / c)
$$

When using a $35 \mathrm{~mm}$ camera with a $35 \mathrm{~mm}$ lens this angle is close to the critical angle of $20^{\circ}$ mentioned earlier. Using a $50 \mathrm{~mm}$ lens the angle is about $15^{\circ}$ and stereoscopic vision is possible, but is strenuous on the eyes. The convergent photograph method, however, is a means of increasing the intersection angle (i) and therefore the depth accuracy when using normal-angle lenses ( $50 \mathrm{~mm}$ for $35 \mathrm{~mm}$ cameras).

\section{Film}

The Kern DSR15 analytical plotter has zoom optics with 5 to 20 times enlargement. In order to take full advantage of the large magnification, a high resolution film should be used for photography. It is recommended that a 64 ASA film is used depending on light conditions. Use colour slide film (the analytical plotter uses film diapositives) and insist that the images are not cut or framed by the film developing company.

Two conflicting factors are considered in the choice of film speed for helicopter or light aircraft operations.
Slow film (low ASA) is needed for high resolution and fast film is desired to reduce image blur from image motion. For helicopter photography in Greenland a 64 ASA high resolution film is often sufficiently fast for photography on a scale of 1:20000 or smaller at a desired shutter speed of 1/250. A faster film (100-200 ASA) should be carried as reserve in case of poor light conditions.

\section{Ground control}

\section{Control points}

Field surveying of ground control points is usually not possible on time constrained geological expeditions. Fortunately, surveyed ground control points are often not necessary to satisfy the accuracy demands of geologic photogrammetry. Control points can be measured with sufficient accuracy on modern topographic maps or stereoscopic vertical or oblique aerial photographs. Points are located that can be identified in both the small-frame photographs and either the map or the aerial photographs. The map is registered to a digitising table or a pen plotter and the coordinates of the located points are digitised. Aerial photographs must be set up and orientated in the analytical plotter and the located points digitised before orientating the small frame photographs. With the multi-model programme both the aerial photographs and several small-frame photographs can be orientated at the same time, which facilitates point identification.

At least three control points are needed for the orientation of a single model. If a block of models is orientated simultaneously, the necessary number of control points averages one per model plus two additional points for the entire block. Identification of reliable points on maps or on vertical aerial photographs that can be transferred to oblique small-frame photographs is often difficult and time consuming. Therefore, any control information obtained in the field that can be used to reduce the need for transferring control points is desirable. Some field observations are easy to obtain. The photographing stations may be identifiable and accurately positioned on a map or on aerial photographs. When photographs are taken from a boat, the height of the camera above water level can be determined. Such information can be added to the block adjustment to improve the orientation, and reduce the demand for transferring control points.

\section{Aerotriangulated points}

Many areas in Greenland are covered by vertical 
aerial photographs on a scale of 1:150 000 taken with a super wide angle photogrammetric camera within the last decade. These photographs are used by GGU for regional topographical and geological mapping and have therefore been aerotriangulated (Hougaard et al., 1991). Using the aerotriangulated points the 1:150 000 photographs are easily set up in the analytical plotter. They are therefore an excellent source of measuring points for the orientation of blocks of small-frame oblique photographs, or of vertical and oblique aerial photographs on a larger scale. The identification, measurement and transfer of points from aerial photographs can be done with an accuracy better than 20 microns on the smallest photograph scale involved. Accuracies obtained in the transfer of points from aerial photographs on a scale of 1:150 000 is therefore better than 3 metres in location and elevation - provided, of course, that the aerotriangulated points used to set up the 1:150 000 photographs have this high degree of accuracy.

\section{Simple field measurements}

Aerotriangulated aerial photographs are a very convenient and efficient source of control points for the setting up of a multi-model block. However, situations may occur where aerial photographs are unavailable, or where they cannot be orientated because of lack of control points or modern maps. Furthermore, they may be on a scale where the point measurement accuracy is insufficient for the actual multi-model mapping project. In such situations control information must be measured in the field. Today, direct measurement of coordinated control points is time consuming and demands surveying equipment and expertise that are expensive to establish. In the near future Global Positioning Systems (GPS) may be able to provide a sufficiently precise and operational solution that can be easily handled.

For the time being, the multi-model method accepts various types of field observations that are especially suited for orientation of close range photographic blocks. The following control types can be used by the multi-model programme: elements such as the camera stations described above, known camera rotations, three-dimensional coordinate points, plane coordinates, elevations, horizontal or spatial distances, coordinate differences, and azimuth measurements. Examples of ground control that can be easily measured in the field are: elevation differences measured with an altimeter, tape- or pace-measured distances along the ground, and compass directions between points that can be identified in the photographs. The multi-model programme is designed so that it is relatively easy for an application programmer to incorporate any new type of control into the bundle block adjustment. Therefore, whatever ground-control information that can be measured in the field should be collected. If the software cannot accommodate the information, a programmer can add modules to the adjustment that incorporates the new type of control.

If the control is based solely on measurements collected in the field, the minimum requirements to orientate a block of photogrammetric models are: one plane position (that may be guessed if the absolute position is unimportant), one pace- or tape-measured distance, one compass direction, and three altimeter elevations. If the block consists of one, two, or three models this minimum is sufficient to ensure reasonable accuracy, although a few additional measurements are strongly recommended to help identify possible gross errors in the control. If the block consists of more than three models, then, in order to control error propagation, the tape distance, compass direction, and altimeter elevations should be repeated for every three or four models.

All control information entered in the multi-model programme is weighted according to a priori estimated errors so that each measurement influences the orientation only according to its own accuracy. Therefore, even inaccurate field observations can supplement the ground control configuration without deforming the photogrammetric tie between models or interfering with other, more accurate, control data. $A$ priori estimates must be determined and supplied by the person who establishes the control in the field.

\section{Summary}

In geologic photogrammetry it is desirable to be able to map from strips or blocks of small-frame photographs taken during field work with a hand-held camera. The combination of vertical or oblique aerial photographs and small-frame field photographs allows detailed mapping in areas of special interest or areas covered with shadows in the vertical photographs.

The method described in this paper has been developed to accommodate these special needs in geologic photogrammetry. A camera calibration procedure is established so that standard small-frame cameras can be used in photogrammetric mapping. The multi-model programme performs photogrammetric orientation of strips or blocks of small-frame photographs, and combines mapping from vertical or oblique aerial photographs with small-frame photographs. The computerguided free movement of the measuring mark between stereo models is a significant aid to photogeologic map compilation, because interpretative work often requires frequent movements between several models of differ- 
ent scales and views to understand and map the geology.

For field photography a small-frame camera with a wide angle lens focused at infinity or with the lens securely fixed at a shorter calibrated focus should be used. In order to obtain the best stereoscopic perception of the model(s), photographs should be taken with camera axes as close to parallel as possible; the convergence angle should be less than $20^{\circ}$. As a precautionary measure, 80 percent photograph overlap should be used rather than 60 percent. High resolution film (64 ASA or slower) is recommended for best results. Although control points are primarily transferred from stereoscopic aerial photographs or topographic maps, simple field measurements can also be used for orientation.

After set up of the photographs in the analytical plotter, software permits the geologist to readily operate the analytical plotter and map from the block of small-frame photographs as easily as from vertical aerial photographs.
Acknowledgements. The multi-model method was developed in part while employed at the Institute of Surveying and Photogrammetry, Lyngby, Denmark supported by the Danish Natural and Technical Science Research Councils, and in part while under contract with the U.S. Geological Survey, Denver, Colorado.

\section{References}

Dueholm, K. S. 1990: Multi-model stereo restitution. Photogrammetric Engineering and Remote Sensing 56(2), 239242.

Dueholm, K. S. \& Coe, J. A. 1989: GEOPROGRAM. Program for geologic photogrammetry. Compass 66(2), 59-64.

Dueholm, K. S. \& Pillmore, C. L. 1989: Computer-assisted geologic photogrammetry. Photogrammetric Engineering and Remote Sensing 55(8), 1191-1196.

Hougaard, G., Jepsen, H. F. \& Neve, J. K. 1991: GGU's photogeological laboratory: aerial photogrammetry - a valuable geological mapping tool in Greenland. Rapp. Grønlands geol. Unders. 152, 32-35.

Pilmore, C. L., Dueholm, K. S., Jepsen, H. F. \& Schuch, C. H. 1981: Computer-assisted photogrammetric mapping system for geologic studies - a progress report. Photogrammetria 36, 159-171. 